\title{
Semi-Empirical Evaluation of the Probability of Structural Rearrangements in Nitrone Spin Traps
}

\author{
F. N. Dultsev ${ }^{1, *}$, G. G. Dultseva ${ }^{2}$ \\ ${ }^{1}$ Institute of Semiconductor Physics, SB RAS, Novosibirsk, Russia \\ ${ }^{2}$ Institute of Chemical Kinetics and Combustion, SB RAS, Novosibirsk, Russia \\ *Corresponding author: fdultsev@isp.nsc.ru
}

Received December 24, 2012; Revised March 25, 2013; Accepted April 07, 2013

\begin{abstract}
Structural rearrangements induced by the action of oxidizers in nitrone spin traps are investigated by means of the semi-empirical calculation of energy changes that accompany the processes assumed on the basis of experimental observations. The mechanisms of cyclization of acyclic nitrones and cycle opening in cyclic dinitrones are assumed on the basis of calculation results. It is shown that water as a solvent plays an important part in these structural rearrangements. Semi-empirical prediction of the reactivity of nitrones as spin traps in oxidative environments is demonstrated to be helpful as a preliminary step to study the spin-trapping capacity of various substituted nitrones.
\end{abstract}

Keywords: spin trapping, semi-empirical simulation, cyclization, nitrone

\section{Introduction}

Spin trapping is a powerful tool to study the mechanisms of chemical reactions by scavending and identifying short-lived intermediates. However, unexpected reactivity of nitrone spin traps toward various oxidizers is a problem for biological or environmental applications of spin trapping [1,2]. To work well as a spin trap, a compound is to capture free radicals but it must not participate in other reactions that might give paramagnetic products. The experimental search for a proper spin trap is a labour-intensive procedure requiring also a deep insight into theoretical organic chemistry. Up to now, no systematic approach has been proposed to simplify this problem. The approach proposed by us involves semiempirical consideration of possible reaction routes and allows theoretical estimation of the reactivity of spin traps both in trapping and in other reactions including the interactions with solvents and with the oxidant components in the systems under investigation. Introduction of definite substituents into nitrones [3] may make these compounds selective to specific kinds of shortlived free radicals; a theoretical investigation of this aspect started with the approach based on frontier orbitals [4]. A much easier theoretical approach reported by us in [5] and applied to spin trapping [6] is now used to consider the possible structural rearrangements that bring complications to conventional spin trapping experiments.

Spin traps investigated in the present work are aldonitrones. The EPR spectrum of a spin adduct of aldonitrone with one $\beta-\mathrm{H}$ atom is a triplet of doublets (six lines as a total); hyperfine splitting constants (hsc) $a_{N}$ and $a_{H}$ depend on the structure of trapped radicals and thus can be used to identify them. Since hsc depend on the density of unpaired electron in the point of the corresponding nucleus, these constants depend on the arrangement of $\mathrm{H}$ $\mathrm{C}-\mathrm{N}-\mathrm{O}$ atoms with respect to each other; due to the higher rigidity of cyclic structures than that of acyclic nitrones, $a_{H}$ for cyclic spin adducts is usually larger and comparable with $a_{N}$ because the hydrogen atom is held closer to the radical centre, while for acylic spin adducts $a_{H}$ is usually 5-10 times smaller than $a_{N}$. The EPR spectrum is often the only basis to assume the structure of spin adducts. An investigation of spin adducts using other methods is often impossible because of the low transformation degrees; one cannot extract the intermediates in the individual form. This is why the necessity of a brief theoretical estimation of the reactivity of spin traps arises.

We showed in $[5,6]$ that semi-empirical calculations of energy changes accompanying the formation of the socalled adsorption complexes can be used to predict the reactivity of compounds. In the present work, we describe an approach based on the semi-empirical evaluation of the side processes that accompany spin trapping reactions. In particular, we focus on the effect of a solvent on possible structural rearrangements of spin traps and their adducts with short-lived free radicals. It is shown that low-level calculations based on semi-empirical methods and molecular mechanics may give adequate explanations of some experimental observations without searching for deep insight into the intimate mechanisms of organic reactions. The proposed approach also allows one to plan the structures of spin traps for specific tasks in various systems.

\section{Experimental}

Short-lived free radicals for spin trapping experiments were generated photolytically [7,8,9]. Photolysis of 
potassium persulphate in water was also used to generate hydroxyl radicals $\left(\mathrm{S}_{2} \mathrm{O}_{8}{ }^{-2} \rightarrow 2 \mathrm{SO}_{4}{ }^{-*}\right.$, followed by $\mathrm{H}_{2} \mathrm{O}+$ $\mathrm{SO}_{4}^{-*} \rightarrow \mathrm{OH}+\mathrm{HSO}_{4}^{-}$).

The EPR spectra were recorded with a BRUKER EMX instrument ( $\mathrm{X}$ band) in a standard flat quartz cell. Microwave power did not exceed $10 \mathrm{mV}$, modulation amplitude was $0.05-0.8 \mathrm{mT}$, other parameters were the same as those listed in [9].

\section{Calculation Procedure}

The interaction of a short-lived free radical with a nitrone was calculated using semiempirical methods MNDO, AM1, PM3, and molecular mechanics MM2. The minimum of the system energy as a function of atomic coordinates was searched for $[10,11]$.

Calculation was based on the idea of a hypothetical adsorption complex (AC) which we consider to precede the formation of reaction products, that is, spin adducts. The AC is formed when a short-lived free radical just approaches the nitrone molecule at a distance several times as long as the expected chemical bond to be formed. Only weak adsorption interactions between the radical and the nitrone exist in the system; no chemical changes occur at that moment. Calculated energy changes accompanying the formation of such an AC allow us to determine the possibility of the formation of expected product; we compare the energies of initial nitrone, $\mathrm{AC}$ and the final product. The stepwise descending positions of these levels are necessary for the reaction to be possible. Other kinds of arrangements usually mean that the expected reaction does not take place.

At first, the structures of the initial nitrone and the spin adduct were optimized using MNDO, AM1 and PM3. Then the structure of the AC was optimized with MM2. CS Chem 3D Pro software was used. The calculation procedure was described in more detail in $[5,6]$.

Nitrone spin traps considered in the present investigation are listed below.<smiles>CC(C)(C=O)N([O-])c1ccccc1</smiles>

1<smiles>CC1=[N+]([O-])C2CCCCC2[N+]([O-])=C1C</smiles>

3<smiles>CC(C)(C)N=Cc1ccccc1</smiles>

5<smiles>CC(=O)C(C)(C)N([O-])c1ccccc1</smiles>

2<smiles>CC1=CN([O-])C2(C)CCCCC2(O)[N+]1[O-]</smiles>

4<smiles></smiles>

Products of side reactions accompanying spin trapping are often diamagnetic, so they cannot be observed with EPR. However, it is particularly interesting when paramagnetic products are formed because these processes may be reasons of mistakes in radical identification. In solutions of acyclic spin traps, sometimes EPR signals with the parameters characteristic of cyclic spin adducts appear, and vice versa. Some observations of this kind were described in [9]. The EPR spectra with unexpectedly large $a_{H}$ appeared in the solutions of nitrones 1 and 2 in the presence of oxidizers. A possible cyclization scheme for compound 1 was attracted to explain the observation [9]; however, no similar routes could be expected for compound 2. Another experimental evidence of changes in the structure of spin traps was also reported in [9]. The EPR signal with small $a_{H}$ appeared in the solution of cyclic dinitrone 4 in the presence of an oxidizer. Table 1 shows the experimental hsc of the spin adducts of nitrones $1-4$.

Table 1. Hyperfine splitting constants of spin adducts (EPR)

\begin{tabular}{|c|c|c|c|c|c|}
\hline \multirow{2}{*}{ Nitrone } & \multirow{2}{*}{ Conditions } & \multicolumn{3}{|c|}{ Observed hsc of adducts, mT } \\
\cline { 3 - 6 } & & \multicolumn{2}{|c|}{ "Cyclic" } & \multicolumn{2}{|c|}{ "Acyclic" } \\
\cline { 3 - 6 } & & $\mathrm{a}_{\mathrm{N}}$ & $\mathrm{a}_{\mathrm{H}}$ & $\mathrm{a}_{\mathrm{N}}$ & $\mathrm{a}_{\mathrm{H}}$ \\
\hline $1-$ acyclic & $30 \mathrm{mM} \mathrm{K}_{2} \mathrm{~S}_{2} \mathrm{O}_{8}, \mathrm{hv}$ & 1.45 & 1.93 & 1.57 & 0.39 \\
\hline 2 - acyclic & $30 \mathrm{mM} \mathrm{K}_{2} \mathrm{~S}_{2} \mathrm{O}_{8}, \mathrm{hv}$ & 1.55 & 1.92 & 1.51 & 0.39 \\
\hline $3-$ cyclic & $5 \% \mathrm{H}_{2} \mathrm{O}_{2}$ & 1.49 & 2.48 & \multicolumn{3}{|c|}{ not observed } \\
\hline $4-$ cyclic & $5 \% \mathrm{H}_{2} \mathrm{O}_{2}$, hv & 1.55 & 1.44 & \multicolumn{1}{|c|}{1.87} & 0.29 \\
\hline
\end{tabular}

In view of these observations, it was necessary to assess the effect of the components present in the systems (first of all the effect of oxidizing agents and of water as a solvent) on the chemical behaviour of spin traps. May the observed changes in hsc values be related to the influence of water or some oxygenated species? To answer this question, we carried out the semi-empirical calculation of possible structural rearrangements focusing on possible complications in spin trapping technique.

The hsc of nitrone spin adducts are proportional to the unpaired electron density in the points of the corresponding nuclei ( $\mathrm{N}$ and $\mathrm{H}$ atoms). The calculated unpaired electron densities $\mathrm{e}_{\mathrm{N}}$ and $\mathrm{e}_{\mathrm{H}}$ for compound 4 in the cyclic form (cycl.) and for the product formed after opening of the cycle (acycl.) are shown in Table 2. For comparison, experimental $a_{N}$ and $a_{H}$ values are listed, too.

Table 2. The unpaired electron density in the points of $N(\mathrm{eN})$ and $\mathrm{H}$ (eH) atom nuclei for nitrone 4

\begin{tabular}{|c|c|c|c|c|}
\hline $\begin{array}{c}\text { Spin adduct } \\
\text { structure }\end{array}$ & $\mathrm{e}_{\mathrm{N}}$ & $\mathrm{e}_{\mathrm{H}}$ & $a_{N}, \mathrm{mT}$ & $a_{H}, \mathrm{mT}$ \\
\hline cycl. & 0.2903 & 0.1451 & 1.55 & 1.87 \\
\hline acycl. & 0.2560 & 0.0677 & 1.44 & 0.29 \\
\hline
\end{tabular}

One can see that a decrease in $a_{H}$ accompanying opening of the cycle is satisfactorily (though only qualitatively) explained by changes in the molecular structure.

Calculation of energy changes accompanying the cyclization of nitrone 1 into a five-membered ring as assumed in [9] showed that the proposed scheme is reasonable. The scheme is presented in Figure 1.

The unshared electron pair of the nitrogen atom is shifted to the corresponding carbon atom, so there is a positive charge $\delta+$ on $\mathrm{N}$, while $\delta$ - appears on $\mathrm{C}$.

Cycling of nitrone 2 proceeds in quite a different manner. The presence of the nitrone group as a substituent in the benzene ring makes the protons in the ortho position to the nitrone substituent more acidic than in benzene 
without substituents. This simplifies the formation of a cycle. The scheme is shown in Figure 2.

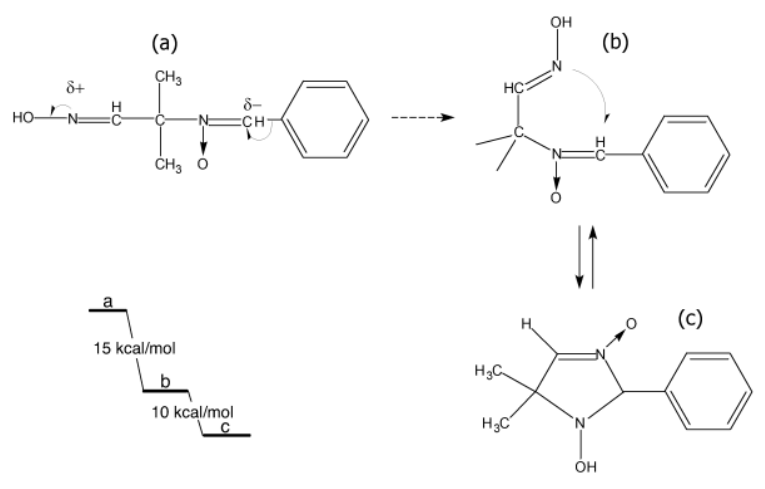

Figure 1. Cyclization scheme for acyclic nitrone 1 and the energy diagram for cyclization with the participation of water: a - initial nitrone, $\mathrm{b}$ - intermediate state, $\mathrm{c}$ - product.

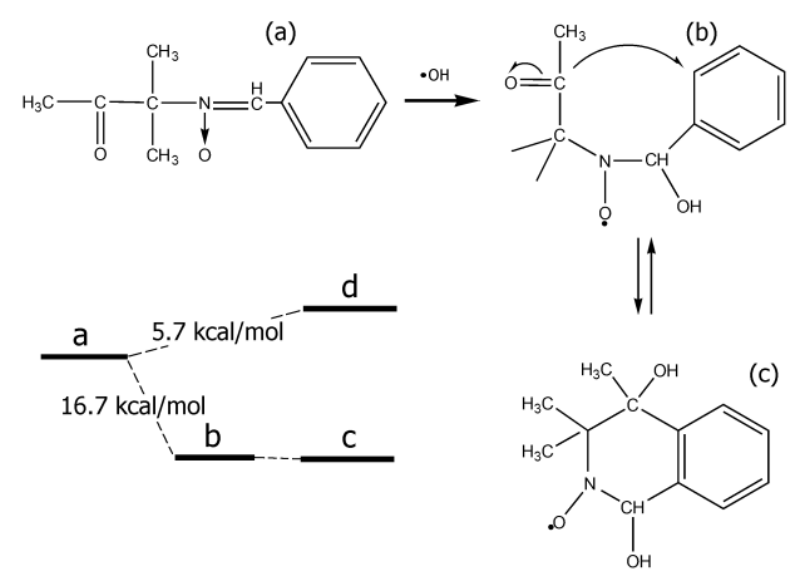

Figure 2. Cyclization of nitrone 2. A scheme and energy diagram: a initial nitrone, $\mathrm{b}$ - its spin adduct with $\mathrm{OH}, \mathrm{c}-$ cyclic product formed from the spin adduct; $\mathrm{d}$ - cyclic product formed from the initial nitrone without trapping $\mathrm{OH}$ radical

\subsection{Modeling Results}

We will consider two routes of cyclization for acyclic nitrones 1 and 2 . The first route starts with nitrone cyclization (without trapping), then the cyclic nitrone traps $\mathrm{OH}$. The second route starts with trapping which results in the formation of $\mathrm{OH}$ adduct, then the acyclic spin adduct undergoes cyclization. Calculating the total energy effects of these two routes and taking into account the role of solvent (water), one may choose which route is favourable from the viewpoint of energy. Calculation results show than the first route is preferable for nitrone 1. For nitrone 2, the second route is more favourable. Since the transformations take place in aqueous solutions, we took into account the role of water in cyclization processes. The energy diagrams of cyclization are shown in Figure 1 and Figure 2 in the left lower part. For nitrone 1, the state $a$ of the energy diagram corresponds to the initial molecule in the presence of water; state $b$ is some intermediate state preceding cyclization with the participation of water molecule that helps the nitrone molecule to coil up; $c$ is the product of cyclization. Total energy effect of the process shown in this scheme is $25 \mathrm{kcal} / \mathrm{mol}$ (for comparison, the route involving $\mathrm{OH}$ trapping by nitrone 1 , followed by cyclization, is characterized by a small positive energy effect, which proves that the first route is preferable for nitrone 1). Nitrone 2 follows the route shown in Figure 2: trapping, then cyclization. One can see in the energy diagram shown in the left lower corner of Figure 2 that the energy effect of $\mathrm{OH}$ trapping is $16.7 \mathrm{kcal} / \mathrm{mol}$. Then cyclization occurs with a small energy gain; the total energy effect is about $18 \mathrm{kcal} / \mathrm{mol}$. State $a$ is the initial nitrone; $b$ is its adduct with $\mathrm{OH}$ coupled with a water molecule before cyclization; $c$ is the cyclic spin adduct. State $d$ corresponds to a cyclization product that would be formed directly from the initial nitrone 2 without trapping $\mathrm{OH}$. One can see that the presence of water makes the route $a \rightarrow b \rightarrow c$ favourable while without trapping $(d)$ the process becomes improbable.

It is the trapped $\mathrm{OH}$ that causes redistribution of the electron density at $\mathrm{N}$ and $\mathrm{C}$ atoms of nitrone 2 (see Figure 2 ). There is an effective negative charge ( $\square$-) in the orthopositions of the benzene ring; this charge promotes cyclization.

To explain the mechanism of cyclization and the effect of solvent, we may consider the reaction to proceed through an intermediate state in which water plays an important part. For nitrone 1, calculation of the AC involving water shows shortering of the distance from $\mathrm{N}$ to $\mathrm{C}$ atom (between which the chemical bond is to be formed) to $3.167 \AA$ as shown in Figure 3. In this case, water molecule simplifies cyclization by helping the nitrone to coil up. Cyclization becomes more favourable from the point of view of energy when water participates in this process.

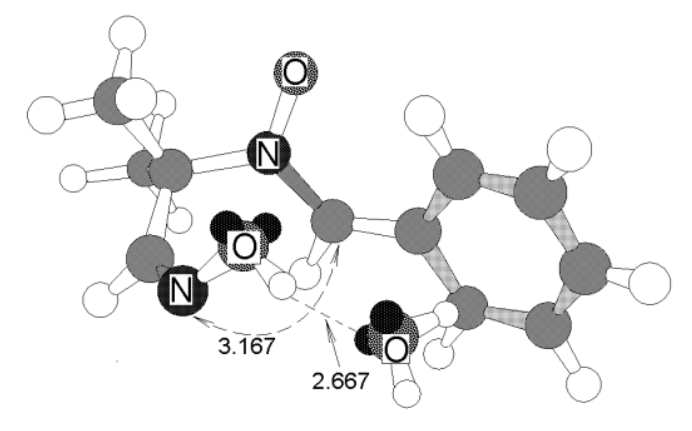

Figure 3. The structure of the adsorption complex during cyclization of nitrone 1 with the participation of water molecule

Indeed, nitrone 1 forms a cycle in the aqueous solution of $\mathrm{K}_{2} \mathrm{~S}_{2} \mathrm{O}_{8}$ both under irradiation and without it, while nitrone 2 forms a cyclic adduct only under irradiation.

\subsubsection{Cycle Opening}

Cyclic nitrones may undergo cycle opening. For example, compound 4 gives rise to a cyclic spin adduct under UV irradiation in $1 \% \mathrm{H}_{2} \mathrm{O}_{2}$ solution $\left(a_{N}=1.55, a_{H}=\right.$ $1.87 \mathrm{mT})$, but at the same time the signal with smaller $a_{H}$ characteristic of acyclic spin adducts appears $\left(a_{N}=1.44\right.$, $a_{H}=0.29 \mathrm{mT}$ ); for compound 3, only the EPR signal with $a_{N}=1.49$ и $a_{H}=2.48 \mathrm{mT}$, which is characteristic of cyclic spin adducts is observed under these conditions; no "acyclic" spin adducts appear [9].

Modeling suggests the scheme of cycle opening reaction for compound 4 as shown in Figure 4. It is the presence of $\mathrm{OH}$ and $\mathrm{CH}_{3}$ groups that promotes cycle opening; this process becomes possible only after the short-lived radical gets trapped. 


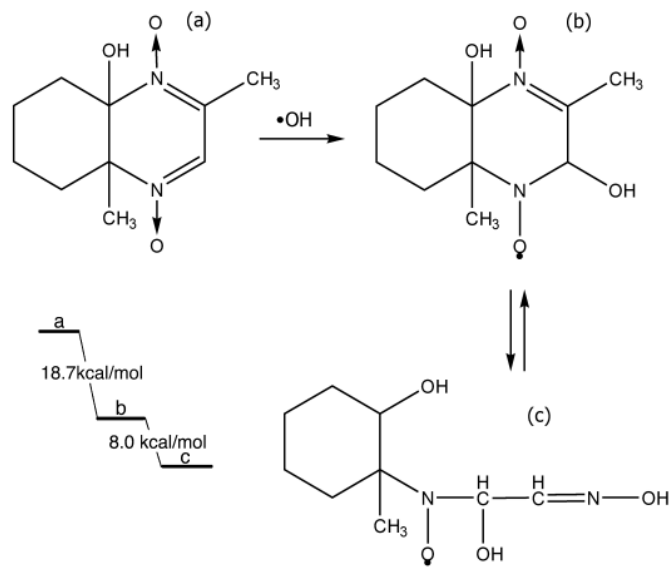

Figure 4. Cycle opening for nitrone 4, with the participation of water (not shown in the scheme), and the energy diagram: $a$ - initial nitrone, $b$ - adsorption complex, $\mathrm{c}$ - product.

After trapping $\mathrm{OH}$, the spin adduct of nitrone 4 undergoes the redistribution of electron density (a shift to the nitrogen atom of the $\mathrm{N}-\mathrm{O}$ group); the hydroxyl group of the nitrone molecule provides the shift of electron density to the carbon atom; the latter thus gets an effective negative charge. Water molecule interacts with the oxygen atom of the $\mathrm{N} \rightarrow \mathrm{O}$ group, which weakens the $\mathrm{C}-\mathrm{N}$ bond and simplifies its rupture. The structure of the $\mathrm{AC}$ for this process is shown in Figure 5.

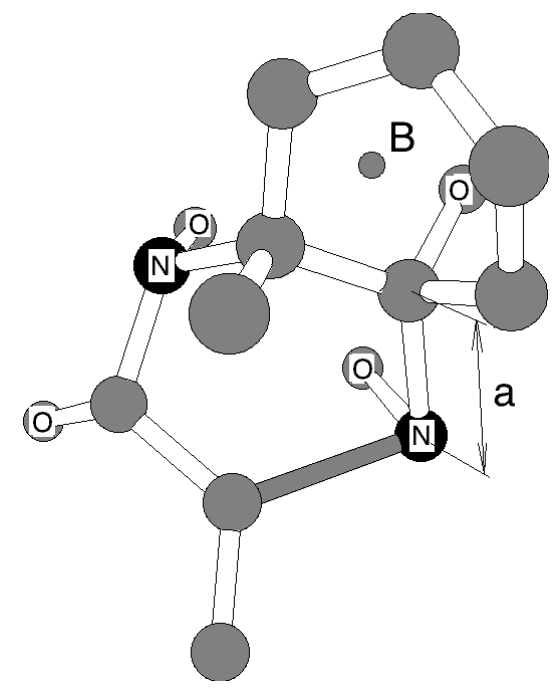

Figure 5. The structure of the adsorption complex during cycle opening for nitrone 4. Hydrogen atoms are not shown. Water molecule (B) promotes bond rupture; the distance (a) between the corresponding $\mathrm{C}$ and $\mathrm{N}$ atoms increases

Hydrogen atoms are omitted. Water molecule is indicated by letter $\mathrm{B}$. This route (through $\mathrm{OH}$ radical trapping) is more favourable from the viewpoint of energy than the version of cycle opening without $\mathrm{OH}$ trapping. Modeling through the AC involving water molecule shows that this process is more favourable (additional energy gain for the case of water participation is $\sim 13 \mathrm{kcal} / \mathrm{mol}$ ). In Figure 5, attention should also be paid to an increase in the length (a) of the bond to be broken in the $\mathrm{AC}$ in the case when water participates in the process (C-N bond length changes from 1.528 to $1.557 \AA$ ). So, it may be stated that water plays an important part in cycle opening reaction. It also affects the stability of the comopunds of this class, which should be taken into account when using this spin trap. A simialr calculation for compound (3) showed that cycle opening is not probable in this compound. The energy diagram of cycle opening reaction for compound 4 in aqueous solution is shown in Figure 4.

It was also interesting to see how semi-empirical predictions correspond to the experimental results reported for known widely used spin traps: $\alpha$-phenyl-Ntertbutylnitrone (PBN, compound 5) and 5,5-dimethyl-1pyrroline-N-oxide (DMPO, compound 6). We estimated the possibility of cyclization or opening of cycle under the action of oxidizers. Modeling showed that for PBN cyclization becomes possible only for spin adduct, that is, after $\mathrm{OH}$ radical is trapped. However, water participates in the additional stabilization of the acyclic structure, so it remains favourable. Thus, $\mathrm{PBN}$ may be considered as a stable spin trap. Indeed, cyclization of PBN in spin trapping reactions has never been reported. We carried out additional experiments with $\mathrm{PBN}$ in the presence of oxidizers generating $\mathrm{OH}$ under UV irradiation (aqueous $\mathrm{H}_{2} \mathrm{O}_{2}$ up to $10 \% ; \mathrm{K}_{2} \mathrm{~S}_{2} \mathrm{O}_{2}$ up to $100 \mathrm{mmol} / \mathrm{l}$ ) and did not observe cyclization.

Modeling of the interaction of DMPO with oxidizers confirms that this compound is not very stable; various side processes can be expected. This gives us ground to warn that the results obtained with DMPO as a spin trap should be interpreted very carefully in view of the high reactivity of this compound in the presence of oxidizers. In addition to oxidation, cycle opening reaction is also possible. Calculation suggests that cycle opening should be expected at the bond between the two methylene carbon atoms, this process being most probable for the oxidized form. We can find some experimental evidence in literature: various complications in interpreting the EPR spectra of DMPO spin adducts were reported (for example, see $[12,13,14])$. We again evidence a contradiction between the desired high sensitivity of a spin trap and the requirement for its stability [15].

\section{Conclusions}

The approach based on semi-empirical calculations of the energies of formation of the adsorption complex and the final products allows one to predict the possibility of side processes accompanying spin trapping, such as cycle opening in the cyclic nitrones or their spin adducts and cyclization of acyclic ones. The proposed approach may be used in theoretical selection of proper spin traps for specific free radical processes. It is shown that a solvent plays an important part in the changes of the structure of adsorption complexes and reaction products, so the influence of a solvent on spin trapping reactions cannot be neglected.

\section{References}

[1] Janzen, E.G., Spin Trapping. Accounts Chem. Res. 4 (1971) 31-40.

[2] Perkins, M.R., Spin Trapping. Adv. Phys. Org. Chem. 17 (1981) 1.

[3] Zubarev, V.E., Spin Trapping. Applications in Chemistry, Biology and Medicine. Moscow, MSU Publishers, 1985.

[4] Fleming, J., Grenzorbitale und Reaktionen organischer erbindungen. N.Y.: Chem.-Verl. 1979. 
[5] Dultsev, F.N., Structure of adsorption complex: Modeling and experiment. Journ. Structur. Chem. 47 (2006) 575.

[6] Dultsev, F.N., Dultseva, G.G. Predictive capacity of semiempirical MNDO/PM3 and molecular mechanics MM2 estimations of the reactivity of cyclic nitrones as spin traps. Chem. Phys. Lett. 429 (2006) 445.

[7] Skubnevskaya, G.I., Dultseva, G.G., Shchukin, G.I., Volodarsky, L.B., Effect of $\mathrm{pH}$ on EPR spectra of the radical adducts of new spin trap 1,2,2,5,5-pentamethyl-3-imidazoline-3-oxide. Izv. AN SSSR ser. khimich. 2 (1987) 312.

[8] Dultseva, G.G., Skubnevskaya, G.I., Volodarsky, L.B., Dulepova, N.V., Tikhonov, A.Ya., New spin traps: 2,3-dihydropyrazine-1,4dioxides - cyclic conjugated $\alpha$-dinitrones and $\alpha$-phenylnitrones with functional groups. Izv. Sib. Otd. Akad. Nauk. Ser. Khim. Nauk, 1 (1989) 77.

[9] Dultseva, G.G., Skubnevskaya, G.I., Tikhonov, A.Ya., Mazhukin, D.G., Volodarsky, L.B., Derivatives of dihydropyrazine-1,4 dioxide, 3-imidazoline 3-oxide and alpha-phenyl nitrones with functional groups as new spin traps in solution and in the gas phase. J. Phys. Chem. 100 (1996) 17523.
[10] Dewar, M.J.S., Thiel, W.J., Ground states of molecules. 38. The MNDO method: approximations and parameters. Amer. Chem. Soc. 99 (1977) 4899.

[11] Dewar, M.J.S., Zoebisch, E.G., Healy, E.F., Stewart, J.J.P., The development and use ofquantum mechanical molecular models. 76 AMI: a new general purpose quantum mechanical molecular model. J. Am. Chem. Soc. 107 (1985) 3902.

[12] Zemel, H., Fessenden, R.W., Electron spin resonance studies of phenyl and pyridyl radicals in aqueous solution. J. Phys. Chem. 79 (1975) 1419.

[13] Harbour, J.R., Chow, V., An Electron Spin Resonance Study of the Spin Adducts of $\mathrm{OH}$ and $\mathrm{HOz}$ Radicals with Nitrones in the Ultraviolet Photolysis of Aqueous Hydrogen Peroxide Solutions. Can. J. Chem. 52 (1974) 3549

[14] Wink, D.A., Desrosiers, M., Unusual spin-trap chemistry for the reaction of hydroxyl radical with the carcinogen $\mathrm{N}$ nitrosodimethylamine. Radiat. Phys. Chem. 38 (1991) 467.

[15] Joule, J.A., Mills, K., Heterocyclic Chemistry. Blackwel Science, Oxford, 2000 